\title{
MAPPINGS IN ASTRODYNAMICS
}

\author{
C. FROESCHLÉ \\ Observatoire de la Côte d'Azur, B.P. 139, F-06003 Nice Cedex, France
}

\begin{abstract}
We review mappings mainly devised for the study of the dynamics of comets and asteroids. An attempt of a typology according to the method used to devise the mapping and to its deterministic or stochastic character is made.
\end{abstract}

\section{Introduction}

Both the existence of kirkwood gaps and the transfer of comets into observable orbits have been the main motivations for building mappings since they have many advantages over numerical integration mainly with respect to computing time and to accuracy. However, even if the brutal force of super computer may in the near future solve these problems, building mappings will still be a challenge to researchers in celestial mechanics. Indeed to build mapping bring a deeper understanding of the general behaviour of non linear dynamical systems.

\section{Deterministic mappings}

\subsection{Analytic MaPPings}

\subsubsection{Wisdom's mappings or Kick's mappings}

The basic idea of the method comes from plasma physics and is due to Chirikov (1979). Wisdom (1982) applied it to asteroid orbit calculations for the $3 / 1$ resonance.

\section{a. Chirikov's method.}

Let us consider the time dependent Hamiltonian

$$
H=I^{2} / 4 \pi+K_{0} / 2 \pi \cos V+\sum_{n \neq 0} K_{n}(I) \cos (V-n t)
$$

where $I$ is the momentum and $V$ its canonically conjugate coordinate. If the constants $K_{n}$ are small then, droping the high frequency terms $\sum_{n \neq 0} K_{n} \cos (V-n t)$, the restricted pendulum Hamiltonian $H_{0}$ gives a good approximation to the system using the averaging principle. However this averaging procedure is no longer valid near the separatrix which is in fact replaced by a narrow chaotic band when the high frequency terms are present. Therefore in order to deal more properly with this problem Chirikov, instead of ignoring the high frequency terms, included them all but in an approximated way, thus obtaining a new Hamiltonian

$$
H_{C}=I^{2} / 4 \pi+K_{0} / 2 \pi \cos V+K_{0} / 2 \pi \sum_{n \neq 0} \cos (V-n t)
$$

which can be considered closer to $H$ than $H_{0}$, since chaos generating high frequency terms are present. 
Using the Fourier transform of the Dirac $\delta$ function with period $2 \pi, H_{C}$ becomes:

$$
H_{C}=I^{2} / 4 \pi+K_{0} \cos V \delta_{2 \pi}(t)
$$

Then by a straightforward integration, using the property that the delta function acts instantaneously, the standard map (see Lichtenberg and Lieberman 1983) is obtained:

$$
\left\{\begin{array}{l}
I^{\prime}=I+K_{0} \sin V \\
V^{\prime}=V+I^{\prime}
\end{array}\right.
$$

\section{b. Wisdom's generalization.}

Wisdom has applied this method to the restricted elliptic three body problem for the $3 / 1$ resonance. From the Hamiltonian derived through a second-order expansion of the perturbing function :

$$
\begin{aligned}
H= & -\mu_{1} / 2 a+H_{\text {secular }}\left(a, e, i, \omega, \Omega, a_{J}, e_{J}, i_{J}, \omega_{J}, \Omega_{J}\right) \\
& +H_{\text {resonant }}\left(---, 3 \lambda_{J}-\lambda,---\right)+H_{\text {highfrequency }}
\end{aligned}
$$

he obtains the new Hamiltonian :

$$
H_{W}=-\mu_{1} / 2 a+H_{s e c}+H_{r e s} \delta_{2 \pi}(t)
$$

from which he derives his mapping. With this, orbits can be computed over millions of years. He found a surprising behaviour : a test particle starting in the gap could remain on a low eccentricity $(<0.05)$ orbit for one million years and then suddenly jumped to a large eccentricity $(>0.3$ ) trajectory, thus becoming a Mars crosser (Wisdom, 1982).

Abrupt changes in orbital behaviour have been known for some time, even before Wisdom work. Scholl and Froeschlé $(1974,1975)$ thought that these were exceptional cases, but Wisdom has shown that such changes are to be expected for any orbit near the $3 / 1$ resonance if one follows an orbit for a sufficiently long period of time. Wisdom's other great achievement was to demonstrate that the observed width of the $3 / 1$ Kirkwood gap coincides with the size of the chaotic region (Wisdom, 1983).

\section{c. A semi analytical interpretation.}

In order to interpret these challenging numerical results Wisdom (1985) developed a semi-analytic perturbation theory for motion near the $3 / 1$ commensurability in the planar restricted three body problem. Three natural time scales are considered : (i) the orbital period (a few years); (ii) the period of libration of the resonant argument (a few hundred years); (iii) the period of motion of the longitude of the perihelion (several thousands years), i.e. the time scale for the slow evolution of the "guiding center" of $\sigma$ and $e$. Taking advantage of these well separated time scales Wisdom approximated analytically the fastest oscillations, i.e. only terms which contain $\sigma$ in the disturbing function were considered. Also terms beyond second 
order in the eccentricity were ignored. Then the very-long-period behaviour was computed numerically under the assumption that the action of the motion on the intermediate time scale is adiabatically conserved during the slow evolution.

The predictions of the theory are in good agreement with the features found on surfaces of section as shown on Figure 1 for orbits generated with his mapping. This figure shows clearly two large chaotic zones. A trajectory in the chaotic zone surrounding the origin enters the narrow part of the chaotic zone which extends to high eccentricity at irregular intervals thus explaining the intermittent bursts of eccentricity. A new criterion for the existence of a large scale chaotic zone is presented and shows that the eccentricity of Jupiter's orbit is at the source of chaos, which confirms the results obtained by Froeschlé and Scholl (1977) using the Schubart averaging procedure.

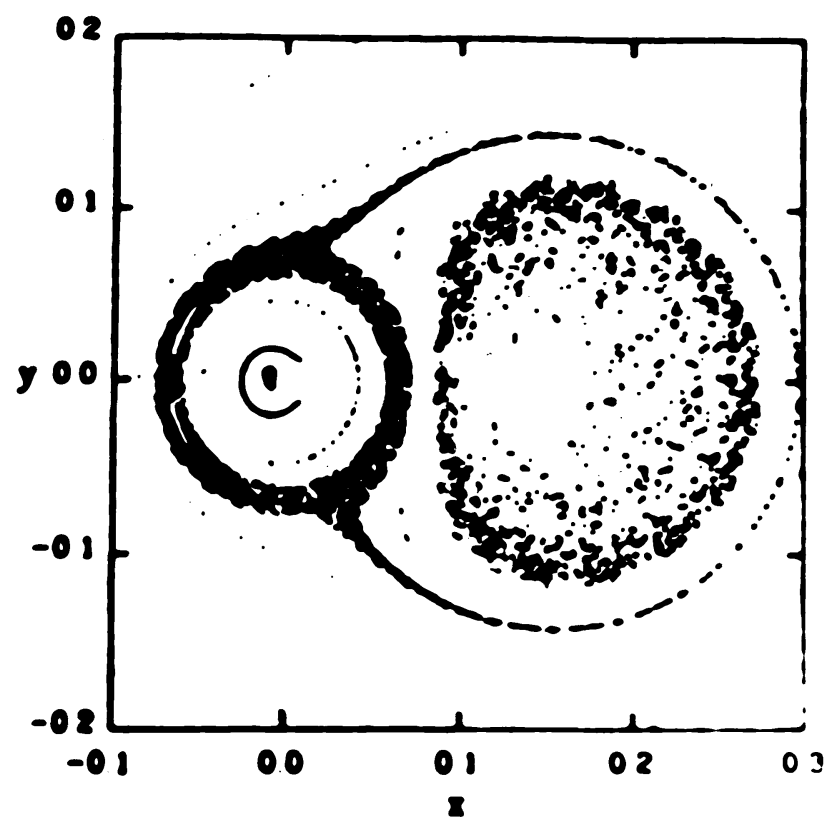

Fig.1: Numerically generated surface of section computed with the Wisdom's mapping. Large chaotic zones appear. The narrow region generates high eccentricities at irregular intervals. From Wisdom (1985).

Of course these results have been checked using different numerical methods. Murray and Fox (1984) have computed the motion of asteroids near the 3/1 resonance using three numerical methods : (a) integration of the full equations of motion, (b) integration of the analytically averaged equation of motion, (c) Wisdom's algebraic mapping. The agreement has been found to be good in the regular regions of phase space. However we have to remark that the higher the eccentricity becomes the less reliable the mapping becomes and therefore after the first increase of eccentricity the dynamical features of the real system may be significantly 
different from the dynamical feature of the mapping.

\section{d. Other Kick's mappings.}

What about the other gaps? The same mapping-methods have been applied by Murray (1986) for the $2 / 1$ and $3 / 2$ Jovian resonances. He determined the chaotic region within these resonances by computing the largest Lyapunov exponent with the rescaling method. He found that both resonances have extensive chaotic regions.

Sidlichovsky and Melendo (1986) obtained similar mappings for the $5 / 2$ resonance. They extended Scholl and Froeschlé (1975) orbits to much longer time intervals (millions of years instead of 38 000) for 96 orbits, and found 53 orbits instead of 33 (Scholl and Froeschlé 1975) for which the eccentricities go beyond 0.3. They thus reproduced the V - shaped nature of the gap. Sidlichovsky (1991) has recently obtained mappings for the third and fourth order asteroidal resonances

Let us come back to Murray's results. The map he derived for the $2 / 1$ and $3 / 2$ resonances includes terms through second order following the earlier derivation of the $3 / 1$ map. The large chaotic zones obtained in both cases are questionable. Indeed the very large eccentricity increases at the $2 / 1$ and $3 / 2$ chaotic zones are artifacts of the truncation of the disturbing function to terms of second order. Henrard and Lemaitre (1987) have shown that a good representation of the motion is only obtained when terms of very high order are included. Actually the miracle of the $3 / 1$ map arises primarily from the fact that as a second order resonance the largest terms in the disturbing function are proportional to two powers of the eccentricities and the next terms are fourth order. An other fortunate and purely combinatorial coincidence is also that the $3 / 1$ resonance is far from other resonances which complicate the dynamics. Anyway it must be emphasized that the failures of the $2 / 1$ and $3 / 2$ mappings were not due to the mapping method itself but to the level of truncation which should not change too much the topology of phase space. Of course an other constraint is that the equations obtained through truncation have to be solvable analytically.

Let us emphasize also that all the dynamics is included in the averaged truncated Hamiltonian and the Kick's mappings can be consider as a first order symplectic integrator of such an Hamiltonian.

\subsection{2. $D Q T$ 's mapping or Hill's method.}

Again in the framework of the restricted circular three-body problem (Sun - planet test particle) a mapping has been derived by Duncan, Quinn and Tremaine (1989) in order to study the long-term evolution of the planetesimal-like orbits in the solar system. As usual the aim is to replace the Hamiltonian dynamical system by an area-preserving mapping that captures much of the relevant physics of the dynamical system. The approximation they use is based on the fact that planets have small eccentricities and the fractional difference in semi-major axes between a near circular orbit of a test particle and the nearest planet is small. Then the perturbations to the motion of a test particle on a near circular orbit are localized near conjunction with the planet then the first-order perturbations to the orbital 
element can be computed analytically using Hill's equations ( Hénon and Petit 1986) or equivalently Gauss' or Lagrange's equations. Away from conjunction the perturbating effect of the planet is assumed to be negligible and hence the orbital elements after conjunction remain unchanged until the next conjunction.

Finally they get a map

$$
\left\{\begin{array}{l}
Z_{n+1}=Z_{n}+\frac{i g \exp \left(i \lambda_{n}\right)}{\varepsilon_{1}^{2}} \operatorname{sgn}\left(\varepsilon_{1}\right) \frac{m}{M_{\odot}} \\
\varepsilon_{n+1}=\varepsilon_{n} \sqrt{1+\frac{4\left(\left|Z_{n+1}\right|^{2}-\left|Z_{n}\right|^{2}\right)}{3 \varepsilon_{n}^{Z}}} \\
\lambda_{n+1}=\lambda_{n}+2 \pi f\left(\varepsilon_{n+1}\right)
\end{array}\right.
$$

where $Z=e \exp (i \tilde{\omega})$ and $f(\varepsilon)=\left|(1+\varepsilon)^{-3 / 2}-1\right|^{-1}, e$ being the eccentricity, $\lambda_{n}$ the longitude of the $n^{t h}$ conjunction and $\tilde{\omega}$ the longitude of periastre and $\varepsilon=\frac{\left|a-a_{P}\right|}{a}$ with $a$ and $a_{P}$ the semi major axes of the particle and of the nearest planet.
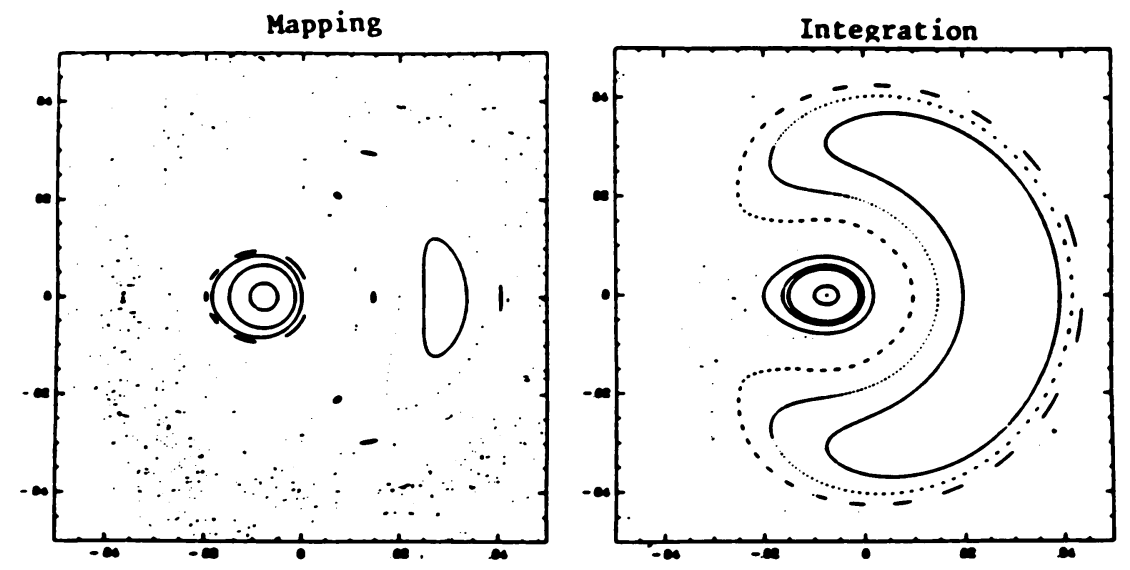

Fig.2: (a) Trajectories of the one-planet map in the complex $y$ planes, for mass ratio $m / M_{\odot}=5.178 \times 10^{-5}$ (Neptune's mass) and dimensionless Jacobi constant $\gamma=8.0694 \times 10^{-5}$. (b) Surface of section for the restricted three-body problem with the same mass ratio and Jacobi constant chosen so that the longitude change of the periodic orbit is the same as in part (a). From Duncan et al. (1989).

Let us introduce the variable $y_{n}=Z_{n} \exp \left(-i \theta_{n}\right)$ where $\theta_{n}=\lambda-\pi f\left(\varepsilon_{n}\right)+\pi$, i.e. the mean longitude of the test particle at the opposition preceding the $n^{\text {th }}$ conjunction. Then Fig. 2 shows orbits of the mapping in the complex $y$ plane and the corresponding surface of section through direct integration.

As the authors state the map correctly reproduces the approximate location, shape and size of the zone of regular orbits near zero eccentricity as well as the 
existence of a larger, second zone of regular orbits centered near $z=0.03$. The map underestimates the size of the second zone but this is not surprising since the approximation $|z| \ll \varepsilon$ is no longer accurate once $|z| \gtrsim 0.03$. The qualitative similarity of the two parts of Figure 2 suggests that the map provides a useful representation of the restricted three-body problem in this parameter regime. They both suggest that there may still be stable bands in the outer solar system containing surviving planetesimals. Let us emphasize that such a model does not take into account secular resonances which certainly play a crucial role for such a problem.

\subsubsection{The Keplerian map or mapping close to a separatrix}

We know that in a neighborhood of any separatrix (i.e. the trajectory with zero frequency of the unperturbed motion) some chaotic motion has to be expected. Actually, the simplest example of separatrix is the parabolic trajectory of the two body problem which separates bounded and unbounded motions. These systems have very long characteristic time scale which allow Petrosky and Broucke (1988) to attack the usual difficulty of small denominators by a new method through Fourier analysis. The system with long time scale can be characterized by "continuous" Fourier spectrum and in this case such a "non integrable system" may become integrable by embedding the small denominator in an analytic function through a suitable analytic continuation. They apply this idea to construct a solution of the restricted three body problem in the case of nearly parabolic motion of the third body and then derive a two-dimensional canonical map which describes the dynamics of long period cometary motion.

Their map called "Keplerian map" reads

$$
\begin{aligned}
P_{n+1} & =P_{n}+2 \sigma \mu s_{1} \sin g_{n} \\
g_{n+1} & =g_{n}-2 \pi \sigma /\left(-P_{n+1}\right)^{3 / 2}
\end{aligned}
$$

where $g_{n}$ is the phase angle of Jupiter when the comet passes its perihelion and $P_{n}$ the energy. $\mu$ is the mass of the small primary, $\sigma= \pm 1$ the parity of the direction of revolution of the particle and $s_{1}$ some scaling energy. Iterations of the Keplerian map show clearly chaotic region confined by KAM torus. Let us remark that the motion near the separatrix of a nonlinear pendulum is much less chaotic (the whisker map). A physical implication of such chaotic motion of comets concerns the structure of a cometary cloud surrounding the solar system: to each parabolic orbit is associated a cloud of comets revolving on elliptic orbits around the Sun with holes and (islands)in the corresponding surface of section. The actual cloud being a superposition of these planar clouds.

A similar mapping related to Keplerian problem has also been proposed by Sagdeev and Zaslavsky (1987)

\subsection{A SEMI-ANALYTICAL APPROACH}

This approach has been pionnered by J. Hadjidemetriou (1991). He develops a new method for the construction of an algebraic mapping that approximates a dynamical 
system which is close to an integrable one. Instead of using the usual method of solving approximately the actual system of differential equations to obtain a discrete system, he starts directly from the integrable system and obtains analytically the corresponding mapping on a suitable surface of section. Then he perturbs this mapping in such a way that it includes all the main features of the non-integrable systems, which are known by analytic and numerical studies.

More precisely starting from an original Hamiltonian $H=H_{0}+\varepsilon H_{1}$ the requirement is to build a mapping with the following properties:

a) It is symplectic.

b) It has the same fixed points as the actual system.

c) The fixed points have the correct stability index.

Actually such a mapping is obtained by a generating function build from the corresponding averaged Hamiltonian and we are back to the problematic of the Kick's mapping and all the method depends on the validity of the averaging procedure, i.e., are the fixed points and their stability index the same for the original Hamiltonian and the truncated averaged one? Therefore, it is not surprising that in the case of the elliptic restricted model starting with the same truncated averaged Hamiltonian Hadjidemetriou has found surfaces of section very similar to Fig. 1 obtained by Wisdom.

\subsection{A SYNTHETIC APPROACH.}

All the mappings described above are ad hoc mappings valid only in some regions of the phase space and for specific purposes. C. Froeschlé and J.M. Petit (1990) have built a mapping valid everywhere in phase space following an idea already used by Varosi et al. (1987) but in the framework of non hamiltonian systems, i.e. systems where attractors do exist.

The basic idea of the synthetic mapping is to interpolate the image of a point, given the images of a set of points, located at the vertices of a grid. The simplest way to achieve this, is to use a linear interpolation (Varosi et al., 1987). Unfortunately, when attractors do not exist, this requires a rather fine graining of the phase space, i.e. we have to compute a lot of points on the grid.

Taylor interpolations appear to be very precise. There exist in any case two key parameters: the number of divisions in each direction $N=$ (total number of cells $)^{(1 / D)}$, where $D(D=2$ in their paper $)$ is the dimension of the surface of section and the order $M$ of the Taylor expansion. In order to explore the validity of the synthetic approach they applied their method for two cases:

1) An algebric area preserving mapping for which the computation of orbits is very fast. This allows to calculate a lot of orbits and to perform enough iterations for a meaningful comparison.

2) A special case of the restricted three body problem studied by Duncan et al. (1989) (see 2 supra) 

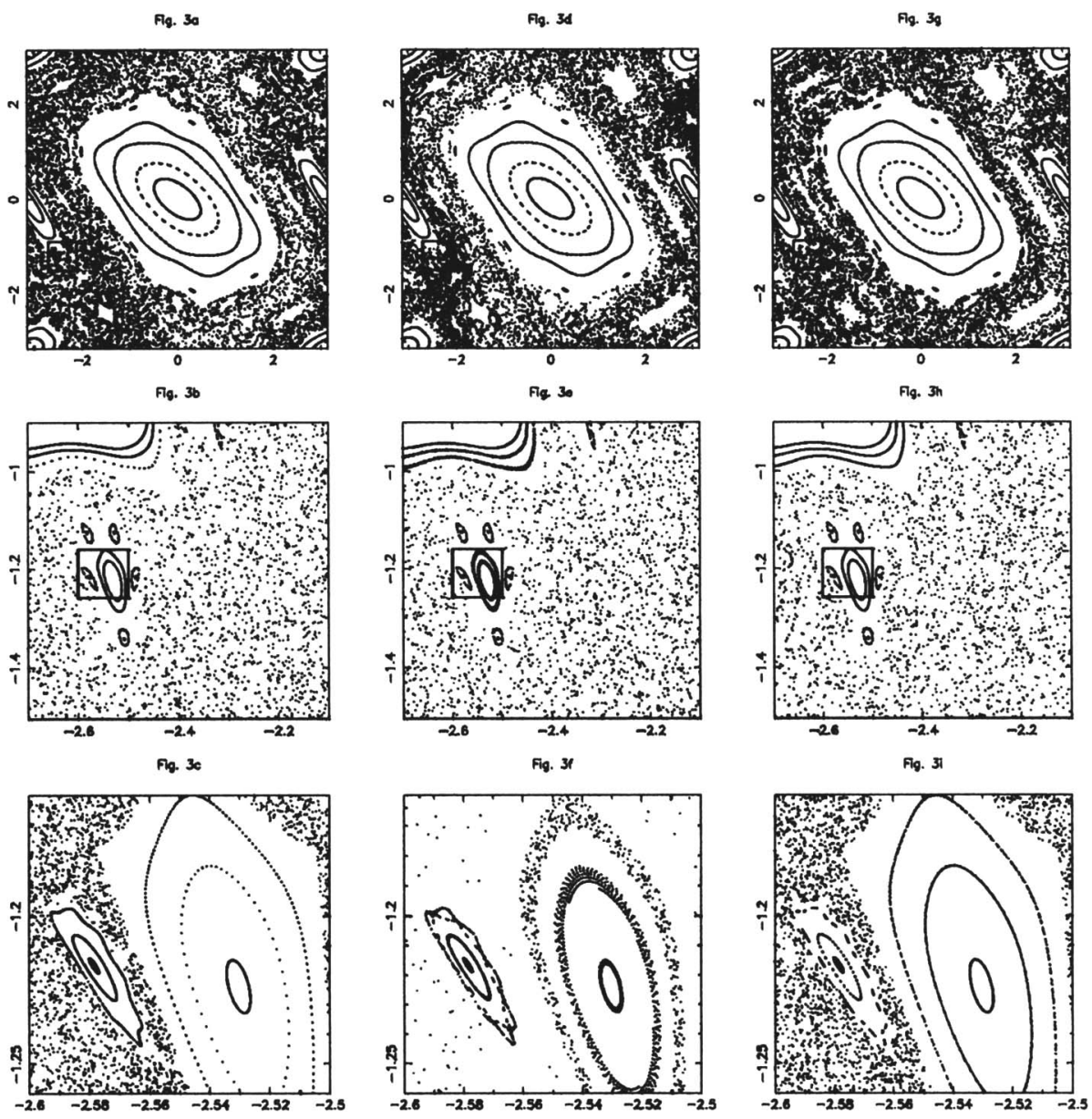

Figs. 3a, b, c: (a) Standard mapping picture for $a=-1.3$ (b) and (c) are enlargements of the small boxes shown in Figs. $3 \mathrm{a}$ and $3 \mathrm{~b}$ respectively. Figs. 3d, e, f: Same as Figs. 3a, b, c for the synthetic mapping T5 and $N=20$.

Figs. 3g, h, i: Same as Figs. 3d, e, f for $N=40$. 
Fie. 40

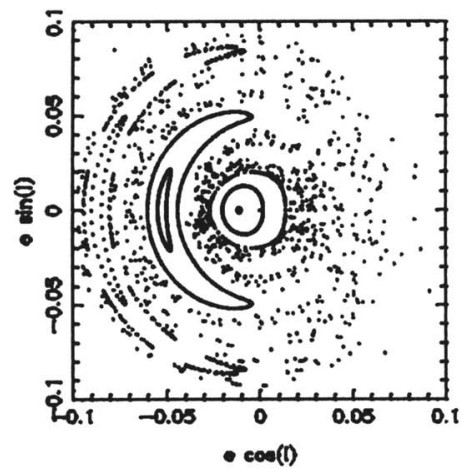

Fig. \&

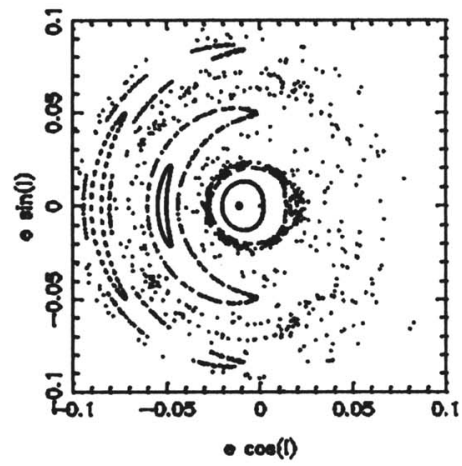

Fig. 40

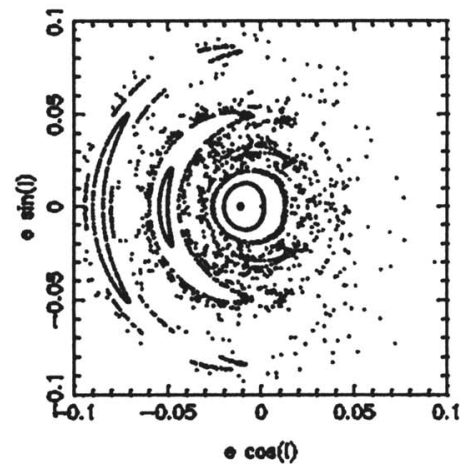

Fie. 46

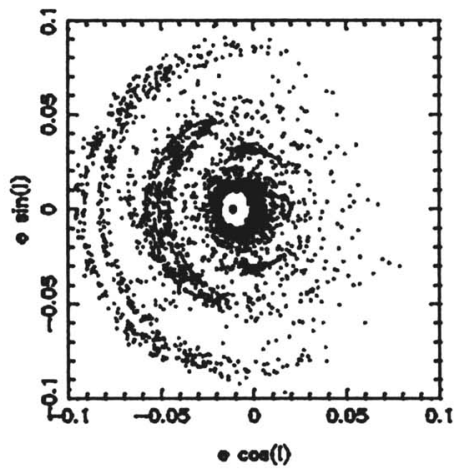

Fing $4 d$

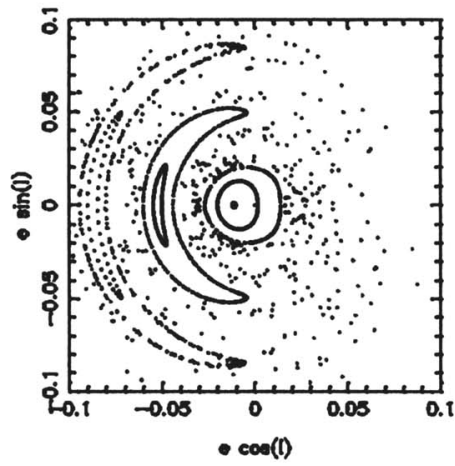

Fig. 4

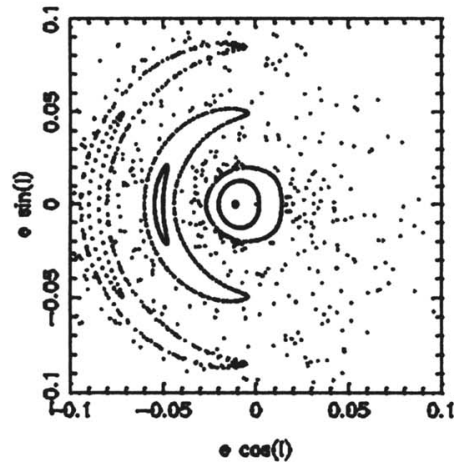

Fig. 4a: Trajectories of the Poincare map of the restricted three body problem, in the plane (eccentricity, mean longitude) at conjunction with the planet, for the Neptune-Sun mass ratio $m / M_{0}=5.17810^{-} 5$ and Jacobi constant 3.0080694 .

Figs. 4b, c, d: Same as Fig. 4a for the synthetic maps T1, T3 and T5. Figs. 4e, f: Same as Figs. $4 \mathrm{~b}$, c for a grid with $N=400$ obtained with T5. 
First the well-known standard mapping has been used. (Froeschlé, 1970; Chirikov, 1979; Lichtenberg and Lieberman, 1983)

$$
\begin{aligned}
& x^{(n+1)}=x^{(n)}+a \sin \left(x^{(n)}+y^{(n)} \quad(\bmod 2 \Pi)\right. \\
& y^{(n+1)} \quad=x^{(n)}+y^{(n)} \quad(\bmod 2 \Pi)
\end{aligned}
$$

Fig.3a shows orbits of the standard mapping for $a=-1.3$, indeed such a mapping exhibits all the characteristics and well-known features of problems with two degrees of freedom such as invariant curves, islands and stochastic zones where the points wander in a chaotic way. Figs. $3 \mathrm{~b}$ and $3 \mathrm{c}$ are magnifications of the small boxes indicated in Figs. $3 \mathrm{a}$ and $3 \mathrm{~b}$ respectively. At this magnification details like second order islands become evident and the approximation qualities of the synthetics maps are easily visualized. Figs. $3 \mathrm{~g}, 3 \mathrm{~h}$ and $3 \mathrm{i}$ correspond respectively to the same orbits and same magnifications as $3 \mathrm{a}, 3 \mathrm{~b}$ and $3 \mathrm{c}$ for the Taylor interpolation mapping in order $M=5$ (T5) where the grid is characterized by $N=40$. We obtain results which look the same as for the original map. When the grid is such as $N=20$ (Figs. 3d, 3e and 3f), it is interesting to notice the agreement of the details in magnifications e and $\mathrm{f}$. This agreement is remarkable since the dimension of the cell (0.31) is much bigger than the dimension of the smallest islands seen in Fig. $3 f$ (0.005). Of course the invariant curve shows some thickness which disappears for $N=40$ but would reappear with a further magnification. This phenomenon is due to the fact that synthetic mapping like T5 does conserve areas only to order 6 .

We have also tested the method on a special case of the restricted three body problem for which Duncan et al. have developed a special mapping. Fig. 4a exhibits orbits of the Poincare map taking as surface of section the plane defined by the eccentricity and the mean longitude as polar coordinates, when the particle is in conjunction with the planet (i.e. in the rotating frame, when $y=0$ and $y^{\prime}>0$ ). On Figs. 4b, c, d are plotted the corresponding orbits for synthetic mapping T1, T3, T5 for a grid with $N=100$. If linear mapping T1 shows only poor qualitative similarities with the Poincaré map, the map T3 correctly reproduces the locations, shapes and sizes of zones of regular orbits. Of course the map T5 is even better. Let us point out that to obtain the same accuracy we needed a smallest grid size (i.e. more points) than for the standard map since the functions which have to be interpolated are less regular. In this case, about 80 minutes were needed to compute the Poincaré map by integrating the equations of motion (Fig.4a) and only 2 seconds for T5. However this should be tempered by the time needed to compute the grid.

Let us remark that the same accuracy than the one obtained by the map T5 can be obtained even for the map T1 by decreasing drastically the grid cell size (i.e. increasing $N$ ). Actually for such an increase of the number of vertices, the price to pay is very low since the additional Poincare return map values at the vertices can be computed using the more precise mapping T5. Figs. 4e, 4f, show the mapping T1 and T3 for a grid with $N=400$ where the $15 / 16$ of the $N^{2}$ map values at the vertices have been computed using T5 through the already computed 
grid with $N=100$. Indeed the accuracy of $\mathrm{T} 1$ is drastically improved. Of course the same holds for T3. This combination of the order of interpolation and of different coarse graining, i.e. of different mappings appears very promising for four dimensional mappings, where the computational time of $\mathrm{T} 5$ is far to be negligible. However Taylor expansion of order 3 and 5 have provided very good results as long we used symmetrical interpolation formulae for which it is necessary to use an extended grid, i.e., a grid extended over the region of the phase space one wants to explore. Since there are cases where one cannot cross a given limit asymmetrical interpolation formulae have been tested and appeared less precise, where the information, within the same accuracy, is concentrated only at the nearest neighbours taking into account gradient information, i.e., not only images of vertices are computed but also the tangential mappings at each vertices. Hence an other type of interpolation has been succesfully used (see Petit J.M. and Froeschlé C.).

\section{Stochastic mappings}

\subsection{Exogeneous stochastic mapping Monte Carlo simulation of stel- LAR PERTURBATIONS (LONG-PERIOD COMETS)}

While it has recently been realized that an important partt of the dynamics of Oort cloud comets arises from regular motion in the Galactic tidal field (Heisler and Tremaine 1986), a decisive role is nonetheless played by individual stellar encounters. Given the physical parameters of such an encounter, its effect on the cometary motion is fully determined. However, the parameters of individual stellar encounters are unpredictable so the stellar perturbations impose a stochastic variation on the cometary orbits.

In Monte Carlo simulations of stellar perturbations (see, e.g., Weissman 1982; Remy and Mignard 1985) the dynamical evolution of a cloud of comets is studied as follows. At a given starting epoch each comet is initialized by choosing a set of orbital elements. These are perturbed by the gravitational effect of passing stars. The geometrical parameters of the stellar encounters are chosen at random. during the passage of a star a comet receives a heliocentric impulse through the interaction of the star with the comet and the Sun. This induces a change in the cometary orbital elements, so these are updated and the comet moves along a new Keplerian ellipse until the next encounter with a random star. In Weissman's procedure the impulse are taken in an even more simplified way from a pre-determined distribution. In other words a stochastic mapping is iterated where the perturbations caused by random stars impose a stochastic process on the cometary orbital elements, which therefore undergo a random walk. It is obvious that all orbits are chaotic and correspondingly the largest LCE is strictly positive. The stochasticity is exogeneous since the stellar encounters occur at random. 


\subsection{Endogeneous STOCHASTIC MAPPINGS (SHORT-PERIOd COMETS AND KuIPER BELT DYNAMICS)}

\subsubsection{Monte Carlo mapping of short-period comet dynamics}

As comets are captured into short-period orbits, i.e. with orbital period of the same order as those of the perturbing planets, the situation changes in a fundamental way. We now have to deal with only the intrinsic stochasticity of a dynamical system which can usually be approximated by a three-body problem: Sun-planetcomet (Rickman and Froeschlé 1988). This stochasticity derives mainly from close encounters with the planet, and during the intervals between such encounters the cometary motion is quasi-regular and predictable. In more precise terms this means that the motion is stochastic only over time scales longer than the typical interval between encounters with the perturbing planet. Thus the phase space domain of short-period comets presents chaotic and ordered regions in an intricate mixture.

Stochastic modelling of this motion is justified by the shadowing principle only if the time step is chosen long enough. Thus we may consider the following procedure (Rickman and Vaghi 1976; Froeschlé and Rickman 1980): we pick a large number of initial cometary orbits at random and integrate these with Jupiter as the perturbing planet over a time interval $\Delta t$, which can be different for different comets. From the resulting set of perturbation we construct stochastic orbital evolutions by picking independently a random perturbation for each successive interval $\Delta t$. For these evolution to simulate real ones we would have a constraint on the choice of $\Delta t$ so that a minimum time step for the random walk could in principle be defined. However,

there are practical problems of such a procedure which are not easy to solve, These are connected with the question of the definition of the perturbation samples: how should one choose the "boxes" of phase space where the initial conditions are to be picked? As already mentioned, the dimension of the boxes can be reduced by symmetry arguments and in the present case a critical point is that short-period comet orbits are known to be generally of low inclinations. One is therefore close to a planar problem with a four-dimensional phase space, and within the limits chosen for the inclination the choice of inclination and orientation of the nodal line is statistically immaterial. Furthermore, since we are dealing with Jupitercrossing or Jupiter-tangent orbits and Jupiter's eccentricity is quite small, there is an approximately circular symmetry such that encounters with the planet can occur with equal probability independent of the orientation of the apsidal line. The choice of the latter is hence also immaterial, and obviously the time-related parameter expressing Jupiter's position at the time of the cometary perihelion passage is the real stochastic variable of the problem which should be taken at random with its true probability distribution. We are left with two orbital parameters which can be taken as, e.g., semi-major axis and eccentricity $(a, e)$, or aphelion and perihelion distances $(Q, q)$.

Boxes in the $(Q, q)$-plane were considered in the Monte Carlo simulations cited above. Let us note that a further reduction of the dimensions appears unfeasible. 
The Tisserand criterion for a co-planar cometary orbit :

$$
T=\frac{2 a J}{Q+q}+2 \sqrt{\frac{2 q q}{a J(Q+q)}}=\text { constant }
$$

might be used to restrict the random walk $(\Delta Q, \Delta q)$ to one-dimensional curves, and the Monte Carlo simulation would then consist of a set of independent, parallel simulations for the differents values of $T$. In each of these one would consider a suitable orbital parameter varying along the curve, such as the inverse semi-major axis $z=1 / a$, and there would be a random walk with step size distribution $f_{i}(\Delta z)$ computed for intervals $\left[z_{i-1}, z_{i}\right]$ along the $z$ axis. However, although small (Froeschle and Rickman 1981), the perturbations $\Delta T$ in the elliptic restricted problem are indeed important for the out-come of the low-velocity encounters with Jupiter occuring in low-eccentricity planet-tangent orbits, and those encounters appear essential for the dynamical transfer of comets.

Quite obviously the number of $(Q, q)$-boxes is limited in practice by the requirement to compute a sample of perturbations large enough to give a fair representation of the dynamics of the region in question. E.g., pronounced non-Gaussian tails are known to exist in the perturbation distributions and the sample must extend far enough into these tails to cover their significant parts. This means that the number of orbits to be integrated in each box may be very large [for a possible way to reduce this problem, see Rickman and Froeschlé (1983)]. At present it thus appears difficult to go much beyond the dynamical resolution of the 30 boxes. On the other hand, this already guarantees a certain "dynamical homogeneity" in the sense that the perturbation distribution over a short interval of time (one orbital period) should not vary too much from one side of a box to the opposite one.

However, within all the boxes we can expect to find smaller regions corresponding to "resonant strips" with $a \approx a_{\text {res }}$, where the comets are close to a simple mean motion resonance with Jupiter. Within such a region there may be slow circulation of the critical argument, in which case it is extremely difficult to define a mean interval between encounters and to find an integration interval $\Delta t$ which is everywhere sufficient. But actually the situation is even worse, because there also exist integrable regions of phase space corresponding to stable librations of the critical argument, where encounters with Jupiter never occur.

The main result of a Monte Carlo simulation of cometary dynamics is a picture of the distribution of comets over the various orbits connected by the stochastic transfer process in question. If the short-period comets are viewed in their most general framework, the dynamics includes perturbations by all the planets as well as nongravitational perturbations, and it would then be interesting to estimate the number of comets trapped in such quasi-stable resonant regions. How this goal would be achieved is not yet clear: the problem is to find an appropriate definition of the perturbation sample boxes and corresponding integration intervals for such a detailed investigation. If on the other hand one considers the dynamical transfer in the three-body problem (Sun-Jupiter-comet), the problem is instead to reach what may be called "topological homogeneity" (Froeschlé and Rickman 1988): Comets 
should then follow only chaotic routes and the relevant boxes from which the initial conditions are to be picked are in fact the intersections of the usual $(Q, q)$-boxes with the chaotic part of phase space. If this restriction is not taken into account, the rate of the transfer is artificially slowed down by the inclusion of irrelevant trappings or an overestimated probability of small perturbations.

\subsubsection{Markov chain modelling}

\section{a. Short-period comet dynamics}

If indeed we consider the orbital distribution of comets as the principal result to be attained, we can again argue that the random walks by individual sample comets considered in the Monte Carlo simulation contain too much information: the only interesting quantity is the number of comets in each $(Q, q)$-box either as a function of time or in a steady state. The same perturbation samples used for the Monte Carlo simulation can then be used to calculate "jump probabilities" between the various $(Q, q)$-boxes over a common time interval $\Delta t$. Defining the state vector $\mathbf{n}$ of the cometary population to be the set of numbers of comets in the different boxes, and calling $P_{i j}$ the jump probability from box $i$ to box $j, \mathbf{n}$ evolves according to a Markov chain:

$$
\mathbf{n}(t+\Delta t)=\mathbf{n}(t) \cdot P
$$

where $P=\left(P_{i j}\right)$ is the transformation matrix. This method was first used in cometary dynamics by Rickman and Froeschlé (1979) for the same domain of the $(Q, q)$-plane as in the above-mentioned Monte Carlo simulation and later on for Oort cloud dynamics by Lago and Cazenave (1983).

This Markov method has the advantage of extreme efficiency, in particular for finding steady-state solution where we have just to solve

$$
\mathbf{n}_{s s}=\mathbf{n}_{s s} \cdot P
$$

i.e., a system of linear equation. Its main disadvantage is that, just like for the Monte Carlo simulation, the number of $(Q, q)$-boxes is limited by the large number of integrated orbits required to obtain accurate estimates of the jump probabilities. If e.g. the 30 boxes of Rickman and Froeschlé are considered, the information obtained on the $(Q, q)$ distribution is strictly limited to the 30 sample points represented in the state vector.

\section{b. The long-term dymamical behaviour of small bodies in the Kuiper belt}

Again, like Rickman and Froeschlé (1979) but in different spirit, in order to study the slow diffusion of small bodies in the Kuiper belt, i.e. to determine the time scales of this process, Levinson (1991) has used Markov chain methods. Here we are not seeking any more steady states solutions but estimates of diffusion times which should be of the order of the age of the solar system.

The $(Q, q)$ plane is also divided into small bins for which transition probability are estimated through sample of 100 particles in each bin which are integrated for 
approximately 100 periods. In addition to the bins within the Kuiper belt which is a transient region two special absorbing bins act as border edges, an inner edge $q<30 \mathrm{AU}$ where the objects become Neptune crossers and an outer edge left as a free parameter with corresponding probability $P_{i i}=1$ and $P_{i j}=0$ for $i \neq j$.

The probabilities matrix takes the form:

$$
P=\left(\begin{array}{ll}
I & O \\
R & Q
\end{array}\right)
$$

and the fundamental matrix

$$
M=\left(M_{i j}\right)=(I-Q)^{-1}
$$

gives the average number of time steps a particle spends in transient bin $j$ before it is absorbed if it is started in $i$. Then $t_{i}=\sum_{j} M_{i j}$ gives the average time a particle spends in all transient bins before absorption. Furthermore, the variance of the particle lifetime is $v=(2 M-I) t-s$ where $s_{i}=t_{i}^{2}$. Finally $f=M R$ gives the probability that a particle starting in transient bin $i$ enter absorbing bin $j$. Using these tools Levinson found that the Kuiper belt is a good candidate as a source of short-period comets. Hovever, in addition to the already discussed possible draw backs of the method (size number of bins, time steps length) the stochasticity underlying the Markov process is indeed very small and may only due to undetected long-period oscillations in the behaviour of $q$ and $Q$.

In the previous study such a low stochasticity was indeed present as an artifact of the method but a strong stochasticity due to strong interactions between the comet and Jupiter was mainly responsible for the diffusion, i.e. big-jumps occured within $\Delta t$ and therefore such an artifact could not invalidate the results.

\section{References}

Duncan, M., Quinn, T., Tremaine, S. (1989) 'The long-term evolution of orbits in the solar system: a mapping approach', Icarus $82,402-418$

Chirikov, B.V. (1979) 'A universal instability of many-dimensional oscilator systems', Phys. Rep. $52,263-379$

Froeschlé, C. (1970) 'A numerical study of the stochasticity of dynamical systems with two degrees of freedom', Astron. Astrophys. 9, 15-23

Froeschlé, C., Scholl, H. (1977) 'A qualitative comparison between the circular and elliptic SunJupiter-Asteroïds problem at commensurabilities', Astron. Astrophys. 57, 33-39

Froeschlé, C., Rickman, H. (1980) 'New Monte Carlo simulations of the orbital evolution of short period comets and comparison with observations', Astron. Astrophys. 82, 183-194

Froeschlé, C., Rickman, H (1981) 'A Monte Carlo Investigation of Jovian Perturbations on ShortPeriod Comet Orbits', Icarus 46, 400-414

Froeschlé, C., Rickman, H. (1988) 'Monte Carlo modeling of cometary dynamics', Celestial Mech. 43, 265-284

Froeschlé. C., Petit, J.M. (1990) 'Polynomial approximations of Poincaré maps for Hamiltonian systems', Astron. Astrophys. 238, 413-423

Hadjidemetriou, J. (1991) 'Mapping models for Hamiltonian systems with applications to resonant Asteroïd motion', preprint

Heisler, J., Tremaine, S. (1986) 'The influence of the galactic tidal field on the Oort comet cloud', Icarus $65,13-26$

Hénon, M., Petit, J.M. (1986) 'Series expansions for encounter-type solutions of Hill's problem', Celestial Mech. 38, 67-100 
Henrard, J., Lemaître, A. (1987) 'A perturbative treatment for the 2/1 jovian resonance', Icarus $69,266-279$

Lago, B., Cazenave, A. (1983) 'Evolution of the cometary perihelion distance in the Oort cloud', Icarus $53,68-83$

Levinson, H.F. (1991) 'The long-term dynamical behaviour of small bodies in the Kuiper belt', Astron. J. Vol 102, $\mathrm{N}^{\circ} 2,787-794$

Lichtenberg, A.J., Lieberman, M.A. (1983), Regular and stochastic motion, Springer Verlag

Murray, C.D., Fox, K. (1984) 'Structure of the 3/1 jovian resonance: a comparison of numerical methods', Icarus 59, 221-233

Murray, C.D. (1986) 'Structure of the $2 / 1$ and $3 / 2$ jovian resonances', Icarus $65,70-82$

Petit, J.M., Froeschlé, C. (1991) 'Polynomial approximations of Poincaré maps for Hamiltonian systems II' submitted to Astron. Astrophys.

Petrosky, T.Y., Broucke, R. (1988) 'Area preserving mappings and deterministic chaos for nearly parabolic motions', Celest. Mech. 42, 53-79

Remy, F., Mignard, F. (1985) 'Dynamical evolution of the Oort cloud I. A Monte Carlo simulation', Icarus 63, 1-19

Rickman, H., Froeschlé C. (1979) 'Orbital evolution of short-period comets treated as a Markov process', Astron. J. 84, 1910-1917

Rickman, H., Froeschlé, C. (1983) 'A Keplerian Method to estimate Perturbations in the Restricted Three-Body Problem', Moon and Planets 28, 69-86

, Rickman, H., Froeschlé, C. (1988) 'Cometary dynamics', Celest. Mech. 43, 243-263

Rickman, H., Vaghi, S. (1976) 'A Monte Carlo simulation of the orbital evolution of comets in the inner planetary region', Astron. Astrophys. 51, 327-342

Sagdeev, R.Z., Zaslavsky, G.M. (1987) 'Stochasticity in the Kepler problem and a model of possible dynamics of comets in the Oort cloud', il Nuovo Cimento, Vol 97, BN2, 119-130

Scholl, H., Froeschlé, C. (1974) 'A teroïdal motion at the 3/1 commensurability', Astron. Astrophys. $33,455-458$

Scholl, H., Froeschlé, C. (1975) 'Asteroïdal motion at teh 5/2, $7 / 3$ and 2/1 resonances', Astron. Astrophys. 42, 457-463

Sidlichovsky, M., Melando, B. (1986) 'Maping for the 5/2 asteroïdal commensurability', Bull. Astron. Inst. Czech. 37, 65-80

Sidlichovsky, M. (1991) 'Mapping for the third and fourth order asteroïdal resonances', Proceeding of the Tokyo Symposium on 'Celestial Mechanics', ed. Kinoshita, H. and Yoshida, H. 38-44

Varosi, F, Gebogi, C., Yorke, J.A. (1987) 'Symplicial approximation of Poincaré maps of differential equations', Phys. Lett. A 124, 59-64

Weissman, P.R. (1982) 'Dynamical history of the Oort cloud' in Comets, (ed. L.L. Wilkening), Univ. Arizona Press, Tucson, 637-658

Wisdom, J. (1982) 'The origin of the Kirkwood gaps: a mapping for asteroïdal motion near the $3 / 1$ commensurability', Astron. J. 87, 577-593

Wisdom, J. (1983) 'Chaotic behaviour and the origin of the 3/1 Kirkwood gap', Icarus 56, 51-74

Wisdom, J. (1985) 'A perturbative treatment of motion near the $3 / 1$ commensurability', Icarus $63,272-289$ 